\title{
Expression of Snail and E-cadherin in Drug-resistant MCF-7/ADM Breast Cancer Cell Strains
}

\author{
Fenglin Cai1,2, Hualin Xiao2, Yuting Sun2, Dongfeng Wang1 and Jinhai Tang3
}

\begin{abstract}
Objective: To investigate the expression of zinc finger transcription factors-Snail and E-cadherin in adriamycin-resistant human breast cancer MCF-7/ADM cells and non-resistant MCF-7 cells.

Study Design: An experimental study.

Place and Duration of Study: The Affiliated Cancer Hospital of Nanjing Medical University and Jiangsu Cancer Hospital, and Jiangsu Institute of Cancer Research, China, from April 2017 to March 2018.

Methodology: Real-time quantitative PCR technology was used to detect the expression levels of Snail mRNA and E-cadherin mRNA in normal breast cells, adriamycin-resistant human breast cancer MCF-7/ADM cells and non-resistant MCF-7 cells. Western blot was used to detect the expression levels of proteins of Snail and E-cadherin in normal breast cells, adriamycin-resistant human breast cancer MCF-7/ADM cells and non-resistant MCF-7 cells.

Results: The expression of Snail mRNA and protein in adriamycin-resistant human breast cancer MCF-7/ADM cells was significantly higher than that in normal breast cells $(p<0.001)$ and non-resistant MCF-7 cells $(p<0.001)$. The expression of E-cadherin mRNA and protein in adriamycin-resistant human breast cancer MCF-7/ADM cells was significantly lower than that in normal breast cells $(p<0.001)$ and non-resistant MCF-7 cells $(p<0.001)$.

Conclusion: Adriamycin-resistant human breast cancer MCF-7/ADM cell strains had high expression of Snail and low expression of E-cadherin. This points out to a new research direction for the targeted therapy of drug-resistant breast cancer cells, and provides clinical guidance for breast cancer therapy and prognosis evaluation.
\end{abstract}

Key Words: Breast cancer, Snail, E-cadherin, MCF-7/ADM cell strain, MCF-7 cell strain, Drug resistance.

\section{INTRODUCTION}

Breast cancer is one of the most common malignant tumors among women, and it seriously threatens the lives of women. Chemotherapy is still an important treatment for breast cancer, but its overall therapeutic effect is still unsatisfactory, which is largely attributable to the development of drug resistance in tumor cells.1,2 Relevant data show that there is a broad range of doxorubicin resistance during chemotherapy, which is also the main reason for the failure of breast cancer doxorubicin treatment. ${ }^{3}$ Therefore, it is particularly important to look for drugs and methods for overcoming drug resistance to doxorubicin in breast cancer. In addition, the primary cause of death in breast cancer patients is not the growth of preinvasive tumors, but the metastasis of tumors. ${ }^{4}$

1 Department of General Surgery, The Affiliated Cancer Hospital of Nanjing Medical University and Jiangsu Cancer Hospital, and Jiangsu Institute of Cancer Research, 210009, China

2 Department of General Surgery, Northern Jiangsu People's Hospital, 225001, China

3 Department of General Surgery, The First Affiliated Hospital, Nanjing Medical University, Nanjing 210029, China

Correspondence: Dr. Jinhai Tang, Department of General Surgery, The First Affiliated Hospital with Nanjing Medical University, Nanjing 210029, China

E-mail:skypk3@163.com

Received: July 02, 2018; Accepted: October 12, 2018
At present, it is considered that epithelial-mesenchymal transition (EMT) and detachment of primary tumor cells are the key steps for the completion of metastasis of tumor cells. ${ }^{5}$ EMT is regulated by a variety of mechanisms. At present, many closely related transcription factors have been discovered. Among these, Snail is the most important type of transcription factor and belongs to the Snail superfamily of transcriptional repressors. ${ }^{6}$ Many studies have shown that in epithelial tumors, the transcription factor Snail induces EMT by binding to Ebox in E-cadherin promoter region and down-regulating E-cadherin expression. Now, there is less research about Snail and E-cadherin in breast cancer.

The aim of this study was to determine the expression of the adriamycin-resistant human breast cancer MCF7/ADM cell strain and the non-resistant MCF-7 cell strain on the zinc finger transcription factor Snail and E-cadherin, in order to provide a reference for overcoming the metastasis of breast cancer and doxorubicin resistance to breast cancer.

\section{METHODOLOGY}

This study was conduct at The Affiliated Cancer Hospital of Nanjing Medical University and Jiangsu Cancer Hospital, and Jiangsu Institute of Cancer Research, China, from April 2017 to March 2018. Normal breast cells, adriamycin-resistant human breast cancer MCF7/ADM cells and non-resistant MCF-7 cells were purchased from Shanghai Meixuan Biotechnology Co., 
Ltd. Normal breast cells, adriamycin-resistant human breast cancer MCF-7/ADM cells and non-resistant MCF-7 cells were cultured in a medium containing $10 \%$ inactivated fetal calf serum, $100 \mu \mathrm{g} / \mathrm{mL}$ penicillin and $0.1 \mathrm{mg} / \mathrm{mL}$ streptomycin, and was incubated in a constant temperature incubator at $37^{\circ} \mathrm{C}$ with $5 \% \mathrm{CO}_{2}$; and the cell growth was observed daily. After the degrees of fusion of cell strains reached $80-90 \%$, passage was conducted. Then they were partially frozen.

RNA extraction and reverse transcription of cells: normal breast cells, adriamycin-resistant human breast cancer MCF-7/ADM cells and non-resistant MCF-7 cells were added to 6-well plates, and the merging of cells was $90-100 \%$. The number of cells in each well is about $1 \times 10^{7}$. After removing the supernatant and washing twice with PBS, $1 \mathrm{~mL}$ TRIZOL reagent was added in per well. Shook well, and digested for 3-5 minutes. The specific operation was performed according to the kit instructions. At room temperature, in $12000 \mathrm{r} / \mathrm{min}$, it was centrifuged for half a minute, and then moved to the RNase-free collection tube. After that, $70 \mu \mathrm{L}$ RNA eluent was added, $10 \mu \mathrm{L}$ RNA sample was electrophoresed on $1 \%$ agar gel, and the integrity of extracted RNA was determined by a gel imaging system. Thereafter, $1 \mu \mathrm{L}$ RNA solution was taken and the nucleic acid content and purity were measured by using a NanoDrop 2000 ultramicro spectrophotometer. Reverse transcription was performed according to the reverse transcription reaction kit instructions. The RT reaction solution was prepared on ice with a total of $20 \mu \mathrm{L}: 1 \mu \mathrm{L}$ PrimeScriptTM RT Enzyme Mix I, 5x PrimeScriptTM Buffer, $1 \mu \mathrm{L}$ OligodT Primer, $1 \mu \mathrm{L}$ Random 6 mers, $1 \mu \mathrm{g}$ RNA. After diluted to $20 \mu \mathrm{L}$ with RNase Free $\mathrm{dH}_{2} \mathrm{O}$, the reverse transcription reaction was performed on a PCR apparatus under the following conditions: $37^{\circ} \mathrm{C}, 15 \mathrm{~min}$ (reverse transcription reaction) and $85^{\circ} \mathrm{C}, 5 \mathrm{~s}$ (deactivate reactions of reverse transcriptase). After the reaction was completed, the reverse-transcribed cDNA was diluted 5-fold and stored at $-20^{\circ} \mathrm{C}$ until use.

Real-time quantitative PCR was used to detect the expression levels of Snail mRNA and E-cadherin mRNA in normal breast cells, adriamycin-resistant human breast cancer MCF-7/ADM cells, and non-resistant MCF-7 cells. The specific method was as follows: constructed a $20 \mu \mathrm{L}$ Real Time-PCR reaction system, including $10 \mu \mathrm{L}$ Premix Ex TaqTM II (2x), $2 \mu \mathrm{L}$ cDNA, 0.4 $\mu \mathrm{L}$ ROX Reference Dye (50x), $0.8 \mu \mathrm{L}$ upstream primer, $0.8 \mu \mathrm{L}$ downstream primer. The $\mathrm{dH}_{2} \mathrm{O}$ was diluted to $20 \mu \mathrm{L}$. The RT reaction solution was added to the Real Time-PCR reaction system, with predegeneration at $95^{\circ} \mathrm{C}$, for 15 seconds, denaturation at $94^{\circ} \mathrm{C}$, for 60 seconds, annealing at $58^{\circ} \mathrm{C}$, for 60 seconds, extension at $72^{\circ} \mathrm{C}$, for 60 seconds, 40 cycles. GAPDH was used as the reference gene in this experiment. The GAPDH primer sequence was: upstream primer 5'-ACTAGGCGC TCACTGTTCTC-3', downstream primer 5'-CGACCAAA
TCCGTTGACTCC-3'. The Snail primer sequence was: upstream primer 5'-CAACCCACTCAGATGTCAA-3', downstream primer 5'-CATAGTTAGTCACACCTCGT-3'. The E-cadherin primer sequence was: upstream primer 5'-ATTCTGATTCTGCTGCTCTTG-3', downstream primer 5'-AGTAGTCATAGTCCTGGTCTT-3'. The expression of the target gene in normal breast cells, adriamycinresistant human breast cancer MCF-7/ADM cells and non-resistant MCF-7 cells was calculated by using 2- $\triangle \triangle$ Ct relative quantitative method.

Western blot technique was used to detect Snail and E-cadherin protein expression levels in normal breast cells, adriamycin-resistant human breast cancer MCF7/ADM cells, and non-resistant MCF-7 cells. The specific method was to add the cells to a 6 -well plate. The number of cells in each well was approximately $1 \times 10^{7}$. Then $150 \mu \mathrm{L}$ mixed cell lysate was added to each well and pipetted several times with a micropipette to allow the lysate to contact the cells completely and lysed it on ice for $30 \mathrm{~min}$. It was placed at $4^{\circ} \mathrm{C}$ low temperature, centrifuged with $12000 \mathrm{r} /$ minute for five minutes, took the supernatant, and stored at $-80^{\circ} \mathrm{C}$ refrigerator. The collected protein sample was mixed with an appropriate amount of 5xSDS-PAGE protein loading buffer, heated in a boiling water bath for 3-5 minutes, and the prepared protein sample was mixed with the buffer solution and added to the SDS-PAGE gel well. After 120 minutes electrophoresis at $100 \mathrm{~V}$, membrane was transferred and blocked at room temperature for 60 minutes. Snail and E-cadherin primary antibodies were added respectively. Membrane was washed three times for 10 minutes each with TBS. Horseradish peroxidase (HRP)labeled second-antibody diluent (1:2000), was added and shaken at room temperature for 60 minutes, membrane-washing with TBS three times each for 10 minutes, substrate addition, color developing, and photography.

SPSS 21.0 software was used for statistical analysis. Data were expressed as mean \pm standard deviation $(\mathrm{x} \pm \mathrm{s})$. One-way variance analysis was used to compare among three groups. SNK method was used to compare between two groups. $\mathrm{P}<0.05$ indicated statistically significant difference.

\section{RESULTS}

The levels of Snail mRNA and E-cadherin mRNA detected by QRT-PCR showed that compared with normal breast cells, Snail mRNA expression in nonadriamycin-resistant human breast cancer MCF-7 cells was enhanced $(p<0.001)$, and E-cadherin mRNA expression was decreased $(p<0.001)$; Snail mRNA expression was high $(p<0.001)$ and $E$-cadherin mRNA was low $(p<0.001)$ in adriamycin-resistant human breast cancer MCF-7/ADM cells. Adriamycin-resistant human breast cancer MCF-7/ADM cells compared with non- 
Table I: Comparison of the expression levels of snail mRNA and E-cadherin mRNA in different cells.

\begin{tabular}{l|c|cc|cc}
\hline Cell lines & \multirow{2}{*}{$\mathrm{n}$} & \multicolumn{2}{|c|}{ Snail mRNA } & \multicolumn{2}{c}{ E-cadherin mRNA } \\
\cline { 3 - 6 } & & Mean \pm SD & p-value & Mean \pm SD & p-value \\
\hline Normal breast cells & 6 & $0.73 \pm 0.16$ & $<0.001$ & $0.86 \pm 0.23$ & $<0.001$ \\
MCF-7 cells & 6 & $1.48 \pm 0.72$ & & $0.57 \pm 0.20$ & \\
MCF-7/ADM cells & 6 & $3.32 \pm 0.45$ & & $0.24 \pm 0.10$ & \\
\hline
\end{tabular}

Table II: Comparison of the expression levels of snail and E-cadherin proteins in different cells.

\begin{tabular}{l|c|cc|cc}
\hline Cell lines & $n$ & \multicolumn{2}{|c|}{$\begin{array}{c}\text { Expression levels of } \\
\text { snail protein }\end{array}$} & \multicolumn{2}{c}{$\begin{array}{c}\text { Expression levels of } \\
\text { E-cadherin protein }\end{array}$} \\
\cline { 3 - 6 } & & Mean \pm SD & p-value & Mean $\pm S D$ & $p$-value \\
\hline Normal breast cells & 6 & $0.82 \pm 0.20$ & $<0.001$ & $0.94 \pm 0.24$ & $<0.001$ \\
MCF-7 cells & 6 & $1.56 \pm 0.74$ & & $0.68 \pm 0.21$ & \\
MCF-7/ADM cells & 6 & $3.44 \pm 0.48$ & & $0.35 \pm 0.12$ & \\
\hline
\end{tabular}

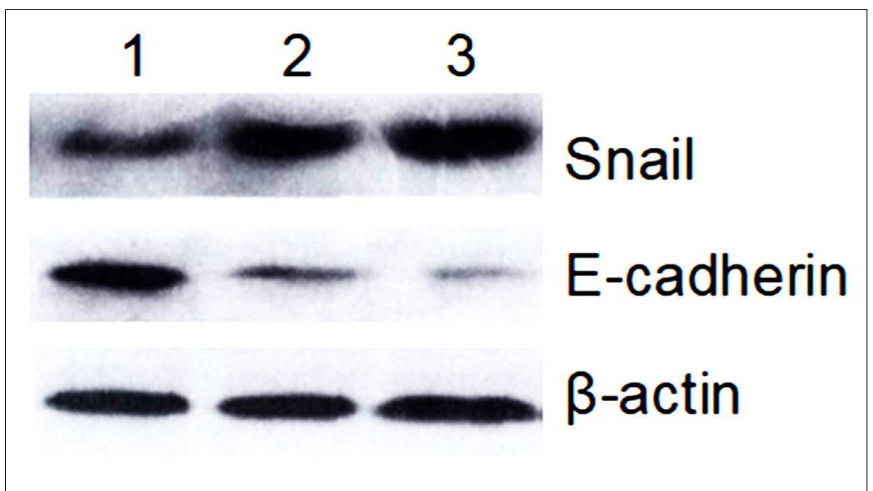

Figure 1: Western blot results of snail and E-cadherin protein expression in different cells.

$1=$ Normal breast cells

$2=$ MCF -7 cells .

$3=$ MCF-7/ADM cells

adriamycin-resistant MCF-7 cells, the expression level of Snail mRNA in resistant MCF-7/ADM cells was significantly higher than that of non-resistant MCF-7 cells $(p<0.001)$, while the expression level of E-cadherin mRNA was weaker than that of non-resistant MCF-7 cells $(p<0.001)$, as shown in Table I.

There were differences in the expression of Snail and E-cadherin protein in different cell strains. The Snail protein expression level of adriamycin-resistant human breast cancer MCF-7/ADM cells was significantly higher than that of normal breast cells $(p<0.001)$, and the protein expression level of E-cadherin was significantly lower than that of normal breast cells $(p<0.001)$. Compared with normal breast cells, the expression level of Snail protein in non-resistant MCF-7 cells increased $(p<0.001)$, and the expression of E-cadherin protein decreased in non-resistant MCF-7 cells $(p<0.001)$. The Snail protein expression level of adriamycin-resistant human breast cancer MCF-7/ADM cells was higher than that of non-resistant MCF-7 cells $(p<0.001)$, while the protein expression level of E-cadherin was lower than that of non-resistant MCF-7 cells $(p<0.001)$, as shown in Table II and Figure 1.

\section{DISCUSSION}

In clinical practice, breast cancer is usually treated by surgery, chemotherapy, radiotherapy, endocrine therapy, biological targeted therapy, etc. Among these, chemotherapy is one of the commonly used methods for breast cancer treatment. ${ }^{7}$ However, chemotherapy resistance is the main reason leading to the recurrence and metastasis of breast cancer patients. 8 The most commonly used agents in chemotherapy is doxorubicin. Due to the resistance of some breast cancer cells to doxorubicin, drug-resistant cells limit the use of doxorubicin. ${ }^{9}$ Drug resistance can produce not only resistance to a chemotherapeutic agents but also crossresistance (namely multidrug resistance (MDR)) to multiple antitumor agents with different structures and mechanisms of action. 10

In recent years, significant progress has been made in the study of drug resistance. The mechanisms of drug resistance mainly include overexpression of drug resistance proteins such as multiple drug resistance protein (MDR1) and breast cancer resistance protein (BCRP), ${ }^{11}$ the changes of some proteases in the cells leading to enhanced cell detoxification, such as overexpression of glutathione-s-transferase (GST) or increased activity, increased ability to repair DNA damage, increased anti-apoptotic ability, and changes in target molecules. However, the mechanism of tumor drug resistance is extremely complex, and the molecular signaling regulatory network caused by tumor drug resistance is still not fully elucidated. Constantly, exploring new mechanisms of tumor drug resistance to improve the efficacy of chemotherapy is one of the key issues to overcome cancer. Studies have shown that chemotherapy resistance is closely related to tumor metastasis. ${ }^{12}$ For example, chemotherapy-resistant tumors are prone to distant metastases, and metastatic tumors have stronger chemoresistance. ${ }^{13}$ This has aroused the wide interest of researchers. Exploring the intrinsic mechanism of drug resistance and metastasis can provide new research ideas for the reversal of tumor resistance and prevention of tumor metastasis during chemotherapy.

The Snail gene superfamily is a family of transcriptional repressors with a zinc finger structure. Snail (Snail1) and Slug (Snail2) are members of the Snail gene superfamily. They are able to bind to a regulatory element of the E-cadherin promoter region, thereby inhibiting E-cadherin expression at the transcriptional level. ${ }^{14}$ Zhang et al. demonstrated that Snail can promote cell motility and vascular formation by regulating the activation of RhoA, which also reveals that Snail may play an important role in tumor growth and proliferation. ${ }^{15}$ Tsubaki et al. found that the receptor activator of nuclear factor- $\kappa B$ (RANK) can activate the $N F-\kappa B$ pathway, thereby enhancing the expression of 
Snail and Twist, and subsequently causing the formation of EMT and migration, invasion and metastasis of tumor cell. 16 In addition, studies have found that comparing the corresponding normal tissue, the expression of E-cadherin in poorly differentiated malignant tumors can be lost completely or reduced, which is a common phenomenon in many human tumors. ${ }^{17} \mathrm{Kremer}$ et al. found that the growth of tumor cells with transfected wild-type E-cadherin was slower than that of control cells, and the expression of E-cadherin was completely lost in tumor metastases. ${ }^{18}$ From the study of esophageal cancer, it was found that E-cadherin negative expression was more prone to hematogenous metastasis, especially liver and lung metastases. ${ }^{19}$

Inhibition of signal molecules that regulate EMT, such as Snail, E-cadherin, and Slug, can increase the sensitivity of tumor cells to chemotherapy drugs. ${ }^{20}$ Although little is known about the molecular mechanism of EMT mediated tumor resistance, there is no doubt that EMT is a common phenomenon in the development of drug resistance. Intervention with EMT can provide a new research strategy for reversing drug resistance in tumor and inhibiting tumor metastasis.

In this experiment, the mRNA and protein contents of Snail and E-cadherin in both adriamycin-resistant human breast cancer MCF-7/ADM cells and nonresistant MCF-7 cells were higher than those in normal breast cells. This further demonstrates that Snail expression is enhanced and E-cadherin expression is decreased in breast cancer cells. This study also showed that Snail protein and mRNA of adriamycinresistant human breast cancer MCF-7/ADM cells were significantly higher than those of normal breast cells and non-resistant MCF-7 cells, and the E-cadherin protein and mRNA of adriamycin-resistant human breast cancer MCF-7/ADM cells were significantly lower than those of normal breast cancer cells and non-drug resistant MCF-7 cells. This shows that Snail and E-cadherin may play a role in the regulation of drug resistance in breast cancer cells, which indicates a new direction for the targeted treatment of resistant breast cancer cells and it has important guiding significance for breast cancer treatment and prognosis evaluation.

\section{CONCLUSION}

In brief, adriamycin-resistant human breast cancer MCF7/ADM cell strains have high expression of Snail and low expression of E-cadherin, which points out a new research direction for the targeted therapy of drug-resistant breast cancer cells, and provides clinical guidance for breast cancer therapy and prognosis evaluation.

Disclosure: This research was supported by the National Key Research and Development Program of China (No. 2016YFC0905900), the "333" Talent Project of Jiangsu Province [No. 4(2016)], the National Key Clinical
Specialist Construction Programs of China [No. 544 (2013)] and Natural Science Foundation of Jiangsu Province (No. BK20151579).

\section{REFERENCES}

1. Li X, Lewis MT, Huang J, Gutierrez C, Osborne CK, Wu MF, et al. Intrinsic resistance of tumorigenic breast cancer cells to chemotherapy. J Nati Cancer Inst 2008; 100:672-9.

2. Goswami S, Wang W, Wyckoff JB, Condeelis JS. Breast cancer cells isolated by chemotaxis from primary tumors show increased survival and resistance to chemotherapy. Cancer Res 2004; 64:7664-7.

3. Steiner R, Stewart JF, Cantwell BM, Minton MJ, Knight RK, Rubens RD. Adriamycin alone or combined with vincristine in the treatment of advanced breast cancer. Eur $J$ Cancer Clin Oncol 1983; 19:1553-7.

4. Ma F, Zhang J, Zhong L, Wang L, Liu Y, Wang Y, et al. Upregulated microRNA-301a in breast cancer promotes tumor metastasis by targeting PTEN and activating $\mathrm{Wnt} / \beta$-catenin signaling. Gene 2013; 535:191-7.

5. Lee JM, Dedhar S, Kalluri R, Thompson EW. The epithelialmesenchymal transition: new insights in signaling, development, and disease. J Cell Biol 2006; 172:973-81.

6. Peinado H, Olmeda D, Cano A. Snail, ZEB and BHLH factors in tumour progression: an alliance against the epithelial phenotype? Nat Rev Cancer 2007; 7:415-28.

7. Chavez-MacGregor M, Gonzalez-Angulo AM. Breast cancer in 2012: New drugs, new knowledge, new targets. Nat Rev Clin Oncol 2013; 10:75-6.

8. Zardavas D, Baselga J, Piccart M. Emerging targeted agents in metastatic breast cancer. Nat Rev Clin Oncol 2013; 10:191-210.

9. Shen H, Wang D, Li L, Yang S, Chen X, Zhou S, et al. MiR-222 promotes drug-resistance of breast cancer cells to adriamycin via modulation of PTEN/Akt/FOXO1 pathway. Gene 2016; 596: 110-8.

10. Nooter K, Stoter G. Molecular mechanisms of multidrug resistance in cancer chemotherapy. Pathol Res Pract 1996; 192:768-80.

11. Burger H, Foekens JA, Look MP, Meijervan MG, Klijn JG, Wiemer EA, et al. RNA expression of breast cancer resistance protein, lung resistance-related protein, multidrug resistanceassociated proteins 1 and 2, and multidrug resistance gene 1 in breast cancer: correlation with chemotherapeutic response. Clin Cancer Res 2003; 9:827-36.

12. Coley HM. Mechanisms and strategies to overcome chemotherapy resistance in metastatic breast cancer. Cancer Treat Rev 2008; 34:378-90.

13. Mccubrey JA, Abrams SL, Fitzgerald TL, Cocco L, Martelli AM, Montalto G, et al. Roles of signaling pathways in drug resistance, cancer initiating cells and cancer progression and metastasis. Adv Biol Regul 2015; 57:75-101.

14. de Herreros AG, Peiró S, Nassour M, Savagner P. Snail family regulation and epithelial mesenchymal transitions in breast cancer progression. J Mammary Gland Biol Neoplasia 2010; 15:135-47.

15. Zhang A, Wang Q, Han Z, Wei HU, Ling XI, Gao Q, et al. Reduced expression of snail decreases breast cancer cell motility by downregulating the expression and inhibiting the activity of RhoA GTPase. Oncol Lett 2013; 6:339-46. 
16. Tsubaki M, Komai M, Fujimoto SI, Itoh T, Imano M, Sakamoto $\mathrm{K}$, et al. Activation of NF-KB by the RANKL/RANK system upregulates snail and twist expressions and induces epithelial-tomesenchymal transition in mammary tumor cell lines. $J$ Exp Clin Cancer Res 2013; 32:62

17. Brabant G, Hoang-Vu C, Cetin Y, Dralle H, Scheumann G, Mölne J, et al. E-cadherin: a differentiation marker in thyroid malignancies. Cancer Res 1993; 53:4987-93.

18. Kremer M, Quintanilla-Martinez L, Fuchs M, GamboaDominguez A, Haye $\mathrm{S}$, Kalthoff $\mathrm{H}$, et al. Influence of tumor- associated e-cadherin mutations on tumorigenicity and metastasis. Carcinogenesis 2003; 24:1879-86.

19. Matsumoto K, Shariat SF, Casella R, Wheeler TM, Slawin KM, Lerner SP. Preoperative plasma soluble E-cadherin predicts metastases to lymph nodes and prognosis in patients undergoing radical cystectomy. J Urol 2003; 170:2248-52.

20. Zhuo WL, Wang Y, Zhuo XL, Zhang YS, Chen ZT. Short interfering RNA directed against TWIST, a novel zinc finger transcription factor, increases A549 cell sensitivity to cisplatin via MAPK/ mitochondrial pathway. Biochem Biophys Res Commun 2008; 369:1098-102.

.......... 